\title{
Relación entre posicionamiento y problematización en la producción escrita de estudiantes de posgrado
}

\section{The relationship between positioning and problematization in the written production of postgraduate students}

DOI: $10.32870 /$ dse.v0i19.500

\author{
María Andrea Vázquez Ahumada*
}

\begin{abstract}
Resumen
La problematización es un momento fundamental en los trabajos de investigación, dado que está estrechamente ligada a la elaboración de objetivos y, en última instancia, a la construcción de conocimiento nuevo a partir de saberes ya planteados dentro de la disciplina en cuestión. En el presente trabajo planteamos que una problematización funcional se relaciona con la forma en que el novel investigador dialoga con las otras voces de la disciplina, cómo se posiciona a sí mismo respecto de las voces de los otros, así como del tipo de contrastes que establece entre los conocimientos previos del tema.
\end{abstract}

Palabras clave: problematización - discurso académico - teoría de la valoración - monoglosia - heteroglosia - posicionamiento.

\begin{abstract}
Problematization is a fundamental moment in research work, since it is closely linked to the develop-ment of objectives and ultimately to the construction of new knowledge based on the knowledge al-ready extant within the discipline in question. This paper suggests that a functional problematization is linked to the way in which novel researchers establish a dialogue with other voices within the disci-pline, how they adopt a position about the voices of the others, and the type of contrasts they make among previous data known about the subject.
\end{abstract}

Key words: problematization - academic discourse - appraisal theory - monoglosia - heteroglossia - positioning.

\section{Introducción}

Este trabajo, cuyo objetivo principal es analizar los recursos empleados para construir la problematización y cómo esta está relacionada con el posicionamiento discursivo de los autores,

* Profesora Investigadora del Posgrado en Ciencias del Lenguaje del Instituto de Ciencias Sociales y Humanidades, "Alfonso Vélez Pliego" de la Benemérita Universidad Autónoma de Puebla (BUAP). Líneas de investigación: Análisis del discurso escolar, discurso académico, Discurso de la ciencia escolar, literacidad en la universidad, literacidad de la ciencia. México.andreina40s@gmail.com 
se centra en el estudio de los protocolos de investigación de alumnos de posgrado. En él se presenta un estudio comparativo de textos escritos por alumnos, en los cuales la construcción del problema de investigación es más funcional en relación con la situación planteada y a la disciplina de las ciencias del lenguaje y protocolos donde la construcción del problema de investigación es menos funcional dificultando el desarrollo de la investigación, el planteamiento de objetivos y, por tanto, la construcción de conocimiento que ello conlleva.

Se presenta en primer lugar una discusión del marco analítico en el que se ubica la investigación. Por un lado, se consideran algunos trabajos hechos en torno de la problematización, la construcción del objeto de estudio y la investigación como elemento fundamental en la construcción del conocimiento (Barton, 1993; Sánchez Puentes, 1993; García Córdoba y García Córdoba, 2005; Pineda, 2011; Torres Carrillo y Jiménez Becerra, 2006). Por otra parte, se hace una revisión del sistema teórico de la valoración (Martin y White, 2005; Hood y Martin, 2007; Hood, 2010), dado el papel crítico que puede desempeñar en la descripción del posicionamiento de los autores al construir un problema de investigación. Particularmente, el trabajo estará centrado en el sistema de compromiso (engagement) (monoglosia y heteroglosia), así como en el de gradación. En este punto se destacará lo planteado por Hood (2010) y Martin y White (2005), quienes han trabajado sobre esta dimensión del compromiso en el discurso académico, sobre todo, en la cuestión de cómo los investigadores-escritores se comprometen con otros cognoscentes y otros conocimientos, a partir de cómo plantean las fuentes de sus posicionamientos y valoraciones. Propongo que la dimensión del compromiso, que, si bien es descrita en los términos anteriores, estará expresada tanto en los elementos léxico-gramaticales de valoración explícita, como en la selección de procesos (verbos) utilizados para introducir las voces de otros autores.

Se debe decir que dentro de los estudios de posgrado la formación de investigadores es un tema central, por lo que nuestra preocupación está enfocada en la cuestión, no solo del conocimiento teórico y metodológico de nuestra disciplina sino en la producción de textos escritos, en tanto que una parte de ser un investigador, efectivamente se relaciona con la escritura. Nuestro planteamiento es entonces que la investigación se constituye, en primera instancia, como la construcción de conocimiento, y una parte de esa construcción es realizada mediante la palabra escrita. Así, el ejercicio de la investigación es un proceso en donde se ponen en juego aprendizajes, saberes y conocimientos orientados a generar nuevas disposiciones para producir nuevo conocimiento. De esta forma se tiene, de entrada, esta doble función de la escritura: construcción de saberes, así como la posibilidad de desarrollar habilidades para comunicar ese saber a una comunidad científica en donde se exponen ideas, se plantean preguntas y se debate.

El presente trabajo se relaciona precisamente con la producción escrita de estudiantes en sus proyectos de investigación orientados a la obtención del grado de Maestría. Particularmen- 
te, este trabajo se focaliza en la cuestión de la problematización, porque, como bien lo plantean distintos teóricos cuando hablan de ella (Barton, 1993; Sánchez Puentes, 1993; García Córdoba y García Córdoba 2005; Pineda 2011), sin problema no hay investigación, dado que este es precisamente el punto inicial de la generación de conocimientos, es el disparador y la guía, el referente durante el proceso de investigación; en última instancia, es lo que el investigador trata de resolver o de averiguar; es lo que busca, lo que explora, lo que quiere explicar. Así, el problema de investigación tiene un papel protagónico durante todo el desarrollo de una actividad de producción de conocimiento en las ciencias, tanto en las así llamadas ciencias duras como en aquellas del ámbito de las humanidades y las ciencias sociales. No solo se trata de describir una situación problemática, sino de construir un problema de investigación que entre en diálogo con la teoría, en tanto que ello permite el avance de la propia investigación, marca un rumbo y señala un vacío que debe ser llenado.

Nuestra propuesta central es, pues, que la problematización efectiva en un texto académico se relaciona por un lado, con el establecimiento de contrastes en la interacción de distintos saberes y aprendizajes sobre el tema de interés, los cuales se pondrán en juego para generar nuevas disposiciones de construcción de un nuevo conocimiento; y por el otro, está vinculada con la postura del propio investigador en ciernes respecto del tema en cuestión y respecto de las voces de otros, es decir, cómo, quien escribe, se posiciona respecto del conocimiento existente para construir el problema que quiere abordar en su investigación.

Así, y dado que se trata de textos escritos que han de ser evaluados por una comunidad académica, este trabajo pretende analizar el cómo se posicionaron los propios autores dentro del debate disciplinar y cómo articularon su posicionamiento de una forma congruente. Una de nuestras preguntas iniciales se relacionó con cómo es que los estudiantes mantienen un buen equilibrio entre lo que podríamos llamar la "objetividad", y el posicionamiento personal al plantear una postura contrastante en sus trabajos de investigación al establecer un punto de vista. Es pertinente decir aquí que cada vez más, dentro de los estudios del discurso académico, se toman en cuenta los aspectos evaluativos en tanto que, de acuerdo con Hyland (2012), estos revelan cómo los autores de un texto intervienen no solo para presentar sus hallazgos sino para evaluar lo que ellos mismos están presentando, y por lo tanto, el establecimiento de un espacio dialógico más o menos abierto o cerrado con sus lectores. En última instancia, esto tendrá una función argumentativa ya que de alguna manera es llevar a tus lectores a ver el asunto como tú mismo lo estás construyendo.

\section{Teoría de la valoración: los sistemas de compromiso y gradación y su relación con la problematización}

La Teoría de la Valoración (TVA), emanada de la lingüística sistémico funcional, ha hecho un desarrollo de la metafunción interpersonal y su instanciación en el nivel de lo léxico-gramatical. 
Este aporte, que ha sido desarrollado principalmente por Martin $(1997 ; 2000 ; 2003)$, White (2000; 2003), Martin y Rose (2003; 2007), Martin y White (2005), Martin y Hood (2007) y Hood (2010), se ocupa de lo subjetivo expresado en el lenguaje, ya sea esté escrito o hablado, mostrando cómo en los textos se construyen comunidades de valores compartidos, mediante "emociones, gustos y valoraciones normativas" (Martin y White, 2005: 1).

Lo que se propone es trasladar la mirada del sistema de Modo, que describiría solo la forma en que se intercambian bienes, servicios y/o información, a una propuesta en la que se observe la negociación de emociones y valoraciones individuales o compartidas (Hood y Martin, 2007). Se toman en cuenta las realizaciones léxicas, al mismo tiempo que las gramaticales, entendiendo que la valoración no se realiza en el discurso directamente sino con la acumulación de recursos lingüísticos (Halliday, 1994; Martin y Rose, 2003; Martin y White, 2005) puestos en juego de manera dialógica (Bajtín, en Martin y White, 2005), esto es, considerando al lector u oyente y posicionándose respecto de lo dicho por sí mismo y por otros.

Los recursos valorativos están divididos en los sistemas de ACTITUD, que abarcan el AFECTO, el JUICIO y la APRECIACIÓN, el COMPROMISO (ENGAGEMENT), y finalmente la GRADACIÓN, que opera en dos ejes: la gradación respecto de la cantidad o intensidad, y aquella que implica una gradación respecto de la prototipicalidad y precisión de los límites de una categoría determinada (2005: 135). Asimismo, se debe decir que existen recursos valorativos inscritos o evocados (explícitos o implícitos).

Para estudiar la problematización en la escritura académica escolarizada proponemos que tanto los recursos de compromiso como los de gradación tienen un papel relevante. El primero permite ver cómo el enunciante se posiciona respecto de lo dicho por sí mismo y por otros, permitiéndonos ver cómo "los hace hablar" en su texto - lo cual también es expresión de dicho posicionamiento-, así como la forma en que posiciona a sus posibles lectores en una relación dialógica. Así, al indagar en el posicionamiento intersubjetivo, se pueden encontrar aseveraciones categóricas que son monoglósicas, en tanto que cierran el espacio dialógico, presentando a la proposición actual como irrefutable, donde no hay alternativas dialógicas y, por tanto, el interlocutor no tiene oportunidad de intervención. Cuando hablamos de GRADACIÓN, hablamos de "mecanismos por los cuales quien habla/escribe 'gradúa' la fuerza de la enunciación o el foco de la categorización mediante los cuales los valores semánticos son identificados." (2005: 94). Opera en estos dos ejes que permiten observar en detalle la conceptualización de lo propuesto. A través del papel dialógico que desempeñan, posibilitan alinearse en mayor/menor grado con los valores y posiciones propuestos por el texto, y por lo tanto, asumir una posición respecto de las comunidades de valores y creencias asociadas con dichas posiciones. 
Figura 1. Recursos de apreciación

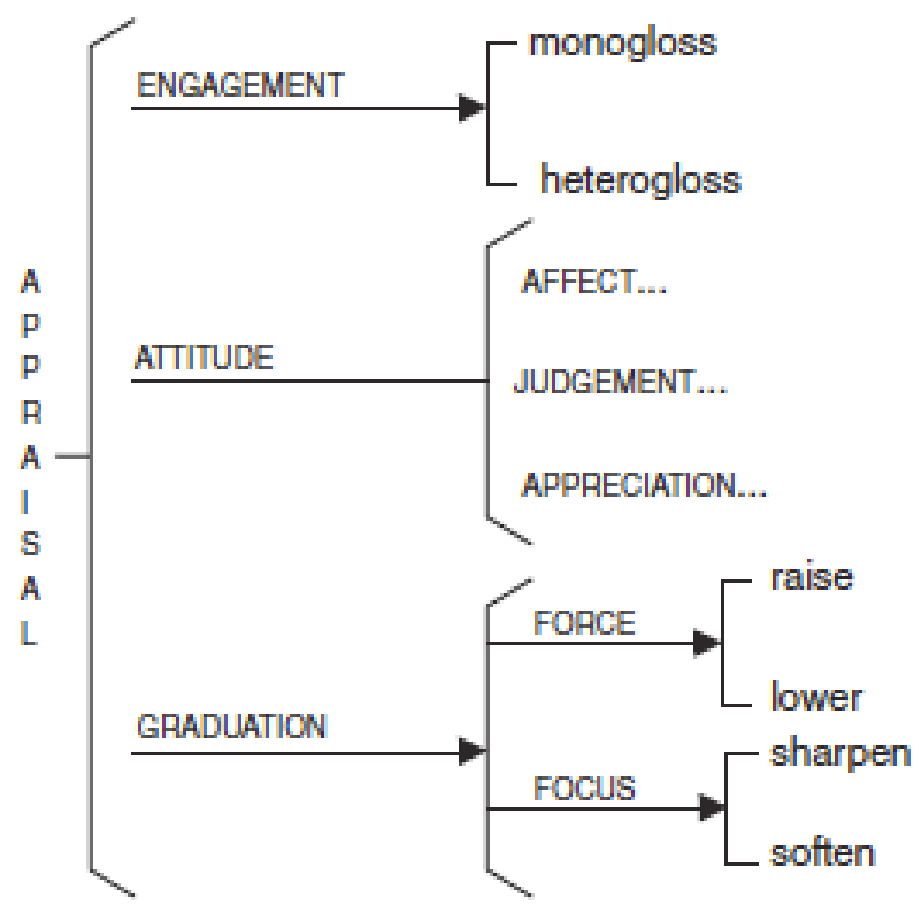

Fuente. Tomado de Martin y White (2005:38)

De esta forma, el marco creado, tanto por la gradación como por el elemento que interactúa con el compromiso, permite determinar el grado de heteroglosia de un texto, así como la forma en que lo planteado está más o menos delineado en términos conceptuales y cómo el grado de la apreciación es "ajustado" (qué tan fuerte o atenuada es la emoción expresada).

\section{Metodología}

A pesar de que solo se abordarán las estrategias de los estudiantes del posgrado en Ciencias del Lenguaje en la problematización de la presentación de sus proyectos de investigación, este trabajo forma parte de una investigación más amplia acerca de la problematización en la formación de noveles investigadores, en la que se pretende describir las estrategias utilizadas por los estudiantes de posgrado en otras áreas del Instituto en Ciencias Sociales y Humanidades de la Benemérita Universidad Autónoma de Puebla, como Sociología, Historia, Estudios Socioterritoriales y Antropología Sociocultural. En el presente trabajo se abordará el análisis de cinco textos distintos de estudiantes de posgrado, en los que se compararán las estrategias de problematización encontradas en los textos que presentaron de manera más o menos efectiva y 
funcional, ${ }^{1}$ en términos de la construcción de un discurso académico que permita el diálogo con la comunidad a la que pretende insertarse.

La manera en que se abordaron estos textos fue desde, por un lado, la Teoría de la Valoración (Martin y White, 2005), emanada de la Lingüística Sistémico Funcional planteada por M. A. K. Halliday $(1985 ;$ 1997) y, por otro lado, los estudios relacionados con el discurso académico y la construcción de identidades, así como otros trabajos relacionados con el análisis del discurso académico, como aquellos realizados por Hunston (1989), Hunston y Thompson (2000), Hyland (2013), Barton (1993) y Martin (2000). Igualmente, se tomaron en cuenta los trabajos de Barton (1993), Sánchez Puentes (1993), García Córdoba y García Córdoba (2005), Pineda (2011), Torres Carrillo y Jiménez Becerra (2006) respecto de la construcción de conocimiento y su relación con la problematización en la investigación.

Así, nos propusimos describir cuáles son los patrones característicos empleados en la problematización, y cuáles son los recursos lingüísticos utilizados en dichos patrones. De acuerdo con Barton (1993), existen recursos de evidencialidad relacionados con la problematización, tales como los recursos de contraste, los recursos de creencias o posicionamiento del autor, así como los de citación y argumentación. Todos estos recursos muestran las estrategias retóricas de la problematización que, desde la teoría de la valoración, pueden ser descritos mediante las categorías de compromiso, gradación, espacio dialógico y contracción o expansión.

\section{Los textos}

Se trata de los escritos donde los estudiantes de la Maestría en Ciencias del Lenguaje presentan sus proyectos de investigación. Dado que en el posgrado se tienen distintas líneas de investigación, ${ }^{2}$ se abordan temas variados que van desde las funciones de cortesía en el lenguaje infantil, la diversidad lingüística, el discurso de la prensa durante las movilizaciones estudiantiles en México en 1968, la función de la música como discurso sonoro en la cinematografía y la construcción discursiva del periodismo independiente.

Aun cuando la investigación incluye más de 20 textos, aquí solo se reportan cinco como una muestra. Tres corresponden a textos en los que la problematización permitió tanto el diálogo con la disciplina y los autores citados, como el posicionamiento claro del investigador/a en ciernes favoreciendo una argumentación más clara y eficaz. Los dos restantes son textos en los que el problema de investigación fue planteado de manera menos eficaz, impidiendo el diálogo con la disciplina y teniendo una argumentación limitada.

1 Es pertinente señalar que esta es una cuestión discutida en la academia del propio posgrado de manera colegiada.

2 Discurso en entornos institucionales; Discurso, narración e intersubjetividad; Discurso y cognición.

Diálo@os sobre Educación año 10 | número 19 | julio-diciembre 2019 | ISSN 2007-2171 


\section{Los recursos de contraste y la contracción o expansión dialógica}

\section{Problematización funcional}

Entre las estrategias de problematización, se observó que los recursos de contraste utilizados en los textos que mejor funcionaron se combinaron con recursos de contracción dialógica, tales como las proclamaciones avaladas (endorsement), que se vieron sostenidas por la citación de autores reconocidos en la materia. Si bien estas citaciones se hacen en ciertos casos tan solo colocando el nombre del autor, el proceso verbal que los sucede es una expresión del posicionamiento del autor del texto ("dice, argumenta, afirma, menciona, sugiere...") permitiendo un sentido de diálogo con dichos autores, es decir, "yo hago esta afirmación con base en lo que otros dicen", y al mismo tiempo con los posibles lectores.

\section{Ejemplo 1:}

Respecto de los recursos de contraste, se ve que este se encuentra entre la diversidad lingüística y la homogeneización lingüística, para lo cual la autora ofrece datos que ilustran dicho contraste:

i) La diversidad lingüística enfrenta amenazas... [PROCLAMA]

ii) Skutnabb-Kangas (2001) asegura que los sistemas educativos, junto con los medios de comunicación, son los agentes más importantes del "asesinato de lenguas"...

iii) Bernstein (2001) afirma que hay una fuerte relación entre educación y control simbólico, control que es necesario ejercer para lograr la homogeneización de todos los grupos que conforman una sociedad... La lengua de un pueblo se relaciona con todos estos aspectos que menciona Karp [PROCLAMA], por ello [CAUSA-CONSECUENCIA] puede [MODULACIÓN] buscarse la homogeneización lingüística dentro de un territorio que interesa construir como un todo.

El contraste aquí descrito se ve reforzado y anclado en los recursos de las proclamas avaladas, que crean un sentido de veracidad, ya que se muestran como innegables y altamente garantizadas (Martin y White, 2005: 126), lo cual se vehicula en la citación a través de los procesos asegura y afirma.

i) La diversidad lingüística enfrenta amenazas que van desde la invisibilidad de una lengua y de sus hablantes (algunas lenguas son más usadas que otras (Phillipson, 1997)); hasta su desaparición (el número de lenguas en el mundo disminuye (Crystal, 2000)).

ii) Skutnabb-Kangas (2001) asegura que los sistemas educativos, junto con los medios de comunicación, son los agentes más importantes del "asesinato de lenguas". Las ideologías, como sistemas de creencias fundamentales de un grupo y sus miembros (van Dijk, 2003), son empleadas a menudo por las instituciones para determinar las pautas a seguir dentro de una sociedad. 
iii) Bernstein (2001) afirma que hay una fuerte relación entre educación y control simbólico, control que es necesario ejercer para lograr la homogeneización de todos los grupos que conforman una sociedad "en todo lo relativo a sus filosofías, sus sentidos comunes y su folklore" (Karp, 1988). La lengua de un pueblo se relaciona con todos estos aspectos que menciona Karp, por ello puede buscarse la homogeneización lingüística dentro de un territorio que interesa construir como un todo.

En estas proclamas avaladas encontramos que la voz autoral se hace responsable de lo dicho, o al menos comparte la responsabilidad con la fuente que se está citando. Aun así, es la autora de este texto quien lleva el peso retórico de lo enunciado. Aun cuando hay una dimensión dialógica entre el autor del texto y las fuentes que está citando, es importante resaltar que, respecto de los lectores del texto, hay una contracción dialógica ya que los procesos que acompañan la mención de los autores (asegura, afirma) son procesos que muestran lo dicho como altamente garantizable, cierran la posibilidad de opiniones alternas, alineando al lector al posicionamiento que la autora está adelantando en su propio texto.

\section{Ejemplo 2}

Aquí también encontramos posicionamientos del autor relacionados con la proclamación. Dicha programación, también, como en el caso anterior, establece un diálogo con los autores que cita. Se debe decir que, en este caso, el posicionamiento del autor no solo está expresado en dichas proclamaciones avaladas, sino en las valoraciones explícitas que hace en forma de apreciaciones sobre el propio trabajo, así como modalizaciones respecto de lo que debe hacerse.

...en el cine, el audio es una herramienta importante para el armado de una historia, ya que permite a los espectadores identificar el ambiente en el que se desarrolla la acción [PROCLAMA].

ii) Antes de describir el problema, es necesario profundizar precisamente en [MODALIZACIÓN DE OBLIGACIÓN; FOCO] el concepto que se acuñó anteriormente, por lo que surgen preguntas realizadas por Armand Belsebre (sin año): “ ¿Existe un lenguaje radiofónico? ¿Tiene la radio un lenguaje específico?", prosigue a responder sus preguntas refiriéndose a uno de los temas centrales [FOCO] que pretende abordar esta investigación: "una parte del pensamiento intelectual que trata el fenómeno de la comunicación de masas como un instrumento de reflexión sobre el proceso de construcción de la realidad que estructuran los medios audiovisuales considera que "cine, sonorización [...] en el sentido de que no disponen de reglas, de un código articulado, no son, estrictamente hablando, unas lenguas"...

iii) El estudioso del lenguaje sonoro reconoce, entonces, la existencia de una estructura sonora que puede, y debe [MODALIZACIÓN], ser reconocida como lenguaje. Esta observación permite que se abra el panorama para tratar la sonorización de una obra cinematográfica como un 
lenguaje $y$, como tal, se presenta al auditorio como una estructura con la capacidad de influir en su percepción y con la inherente facultad de la comunicación para transmitir mensajes de diversos tipos utilizando el mismo canal de transmisión: el sonido.

iv) Una vez habiendo identificado el lenguaje sonoro como tal, surge el problema de establecer si la sonorización imprime valor narrativo o es un recurso para lograr concordancia cognitiva en la descripción del contexto en el que se llevan a cabo los acontecimientos contados dentro de la diégesis. [PROBLEMA DE INVESTIGACIÓN].

Al igual que en el primer caso, hay una estrategia de contraste que permite la problematización ya expuesta:

i) "una parte del pensamiento intelectual que trata el fenómeno de la comunicación de masas como un instrumento de reflexión sobre el proceso de construcción de la realidad que estructuran los medios audiovisuales considera que "cine, sonorización [...] en el sentido de que no disponen de reglas, de un código articulado, no son, estrictamente hablando, unas lenguas"...

ii) El estudioso del lenguaje sonoro reconoce, entonces, de una estructura sonora que puede, y debe, ser reconocida como lenguaje.

\section{Ejemplo 3}

En el siguiente ejemplo, la estrategia de problematización elegida por el autor está basada en: i) hacer una proclama avalada que incluye una valoración que en sí misma ya es un contraste "es diferente". Dicha proclama termina haciendo referencia (aunque débil) a diversos autores; y ii) se refuerza el contraste ya planteado con la locación adverbial A pesar de... y el adversativo, pero.

i) El discurso de la prensa independiente es diferente [PROCLAMA; VALORACIÓN, CONTRASTE] de la prensa en general en tanto que, con base en el ejercicio de los derechos constitucionales de libertad de expresión, pretende generar conciencia sobre aspectos de importancia para la sociedad a manera de noticias, con el fin de lograr una respuesta por parte de su audiencia... El periodismo de investigación tiene una actividad explícita, la investigación. Distintos autores argumentan que la investigación debería ser el principio guía de cualquier noticia (Spark, 2003; García Márquez, 1996; Hunter, 2011, entre otros).

ii) A pesar de la parcialidad de las empresas editoras de noticias y de los mismos periodistas, el periodismo de investigación se propone como un medio para la investigación a fondo de distintas hipótesis sobre un evento, individuo o institución. Lee Hunter, periodista fundador de la Global Investigative Journalism Network, expone la idea de investigación basada en hipótesis, donde se propone que las historias son solamente "the cement which holds every step of the investigative process" (2011: 1), una historia no es más que una hipótesis hasta ser verificada, con 
lo que el proceso de investigación ayuda a legitimarla con base en evidencias. Hunter también señala que el periodismo de investigación busca exponer al público asuntos que son ocultados, que bien puede ser intencional, o por una masa de datos (qué bien puede ser intencional) y circunstancias que oscurecen su entendimiento (2011:7). Sobre esto cabe añadir que el periodismo convencional reporta información otorgada por otras instancias (policía, compañías, gobiernos, etc.), pero el periodismo de investigación busca la información por sus propios métodos, en ocasiones por medio de búsqueda de información en fuentes confidenciales, lo cual (hace) la diferencia de la prensa convencional en tanto que ésta última no necesariamente hará una investigación sobre información que considera material para noticias.

Nuevamente aparecen las citas a un autor reconocido en el campo. En este caso es interesante observar que el tipo de procesos que acompañan dichas citas; i.e. argumentan, expone, se propone que, señala, construyen un cambio en los recursos de compromiso, planteando una atribución más que una proclama, lo cual expande el espacio dialógico, estableciendo no solo un diálogo con el autor citado, sino con la propia audiencia, lo cual plantea la posibilidad de formar parte de una comunidad académica.

\section{Problematización no-funcional}

Los dos textos que a continuación se analizan, presentaron una problematización poco o nada funcional en términos de establecer de manera clara, tanto el asunto a investigar como el posicionamiento del autor dentro de un diálogo con las fuentes citadas y la comunidad a la que pretende insertarse.

\section{Ejemplo 1}

En este texto la autora utiliza no solo una, sino dos estrategias de contraste. La primera se relaciona con la manera en que se plantea un análisis del discurso de textos aislados y la posibilidad de analizar textos en donde se vea el texto producido por alguien y la respuesta a este, es decir, en términos de un diálogo dentro del conflicto social. La segunda estrategia de contraste se relaciona con el propio ejercicio de la disciplina en donde, por un lado, el conflicto mismo de México en 1968 se ha trabajado mucho desde la historia y la sociología, pero muy poco desde el análisis del discurso.

i) En ocasiones, se manejan textos aislados, sin permitir el diálogo entre un discurso enunciado y la respuesta a este.

ii) En el caso de la movilización social de 1968 en México, se ha trabajado mucho desde la Historia (y algo desde la Sociología) y poco desde el Análisis del Discurso, por lo que se tiene un campo inexplorado en el estudio de los conflictos sociales, políticos y económicos, una aproximación a la reconstrucción temporal del acontecimiento... 
iii) Igualmente, tener una muestra de los textos producidos durante la movilización de 1968 permite estudiar cómo se construye una gramática del conflicto; aproximándose a la manera en que los actores del enfrentamiento utilizan recursos lingüísticos para la proyección de su imagen social, la adhesión de colectivos, el apoyo de ciertos sectores sociales y cómo se configuró el proceso histórico por medio de lo que se decía en las calles y desde el mismo gobierno a través de la prensa.

En este ejemplo, si bien la estrategia de contraste está presente, y la problematización pudiera ser funcional dentro de la disciplina, hay una cuestión que la hace fallida ya que la autora no toma en consideración, o no establece ningún tipo de diálogo con fuentes externas a su propia aseveración. En este caso, más que una proclama, lo que tenemos es una aseveración "tajante" - a bare assertion - en donde el espacio dialógico se cierra completamente, lo cual no permite que se entre en contacto con otras voces y otros saberes disciplinares. Casi tendría que ser un acto de fe aceptar lo que la investigadora propone.

\section{Ejemplo 2}

En este caso, la autora no plantea ninguna situación de contraste. En el fragmento i) se observa el uso del condicional si creando una proyección que regula o ajusta las expectativas del lector respecto de lo que habrá de plantear más adelante en su texto: "son los infantes quienes experimentan con el sistema de comunicación y quienes deciden jugar con la gramática y las convenciones para mantener vivo el lenguaje...", lo cual es una aseveración categórica, completamente monoglósica (obsérvese el proceso relacional en presente simple 'son') que se remite únicamente al dicho de la autora.

También los fragmentos ii) y iii) muestran aseveraciones que son dialógicamente contractivas, aunque no completamente monoglósicas, en tanto que tienen modulaciones tales como lleva a suponer y da cabida, introduciendo el propósito de su investigación: "análisis de cómo los padres de familia construyen la identidad de sus hijos y su papel propio dentro de la familia mediante la percepción y promoción del uso de groserías y de cortesía lingüística por parte de sus hijos."

La siguiente valoración (que implica posicionamiento por parte de la autora) "juega un papel importante", nos muestra el interés de la autora más en una cuestión de valores morales, lo cual, si bien no es cuestionable, sí construye una funcionalidad fallida en términos del diálogo que se espera construya con la disciplina y la comunidad académica. Fijémonos en los ítems léxicos que integran su aseveración: 'patrones de socialización', 'comportamientos', 'normas sociales', 'normas de cortesía'... enmarcando su 'problema' no en una cuestión lingüística como se esperaría, sino'moral'y del comportamiento correcto. Efectivamente, habla del uso de groserías y cortesía lingüística, pero sus valoraciones y posicionamientos se encuentran en el marco ya descrito. 
i) ...si ha de haber algún cambio en la lengua, éste es, en algunos casos, iniciado por los niños y no por los adultos pues son los infantes quienes experimentan con el sistema de comunicación y quienes deciden jugar con la gramática y las convenciones para mantener vivo el lenguaje...

ii) Estas diferencias en el desarrollo de las ciudades y del flujo de personas que tiene lugar tanto en Puebla como en Celaya lleva a suponer el uso diferente del lenguaje en la población en general, sin embargo, también da cabida al análisis de cómo los padres de familia construyen la identidad de sus hijos y su papel propio dentro de la familia mediante la percepción y promoción del uso de groserías y de cortesía lingüística por parte de sus hijos.

iii) Esta transmisión de los patrones de socialización juega un papel importante en el desarrollo de la identidad del niño y parte de los patrones que se han [MODALIZACIÓN DEÓNTICA] de transmitir son los comportamientos deseados y los valores tales como la responsabilidad, el amor a la patria, la honestidad, la cortesía y el uso adecuado del lenguaje.

iv) La cortesía o en particular la cortesía lingüística la podemos relacionar con las normas sociales, estas normas dependen del contexto por lo que las normas de cortesía pueden diferir bastante de familia a familia y pueden existir algunas situaciones en las que los comportamientos comunicativos no estén sujetos a las normas de cortesía, situaciones en las que un determinado comportamiento que podría ser visto como descortés sea aceptable; a menudo dichas situaciones están caracterizadas por una gran diferencia de poder (Melorose, Perroy, \& Careas, 2015).

\section{Conclusiones}

De los análisis aquí planteados, podemos decir que se encontraron elementos que permiten una problematización más funcional en un proyecto de investigación en alumnos de posgrado.

Por un lado, los recursos lingüísticos asociados a una problematización mejor elaborada fueron aquellos relacionados con la elaboración de estrategias de contraste, tales como elementos léxicos propios del campo (diversidad vs homogeneización), elementos de negación (no) o de contraste propiamente dichos ( $A$ pesar de, pero). Este tipo de elementos no se encontraron en los textos menos funcionales, lo cual nos lleva a pensar en lo planteado en un inicio: no solo se trata de los elementos de contraste por sí mismos, sino de la puesta en juego de conceptualizaciones y saberes disciplinares, así como concepciones del mundo que se contraponen o tienen alguna carencia que es puesta de relieve, a través del discurso, por parte del novel investigador.

Como ya se mencionó, dado que la estrategia de contraste no basta por sí misma, al mismo tiempo se encontró que la segunda estrategia empleada en los textos más funcionales se relacionó con el posicionamiento de los autores al plantear dichos contrastes. En los textos analizados se encontró la utilización de la citación de autores para, por medio de las proclamas avaladas, y aunque estas proclamas son aparentemente cerradas en términos dialógicos, lo ob-

Diálo pos 
servado es que mediante la citación se permitió construir un diálogo con la disciplina y los autores citados, dando así validez a lo planteado y alineándose con una comunidad de saberes determinada. En el caso del último texto analizado, los recursos de compromiso o engagement utilizados por el autor al citar a los distintos autores fueron de atribución, construyendo un espacio dialógicamente abierto con los propios autores, pero sobre todo con la disciplina.

De los textos menos funcionales, lo obtenido a través del análisis nos señala dos aspectos relevantes. Por una parte, aunque en un texto sí se usó la estrategia de contraste y en otra no, en ambos textos las autoras construyeron sus discursos de manera monoglósica, haciendo aseveraciones 'tajantes' o rotundas - bare assertions - las cuales no dejan cabida a opiniones de ninguna índole, es decir, no en contra pero tampoco a favor. Hubo una ausencia de autores citados, lo cual no construyó un diálogo ni con la disciplina ni con la comunidad en la que se insertarán.

A partir de lo aquí expuesto, se propone que en trabajos con alumnos acerca de escritura académica se insista en una problematización vinculada a situaciones semejantes, ubicándola dentro del contexto teórico y disciplinar y no en un contexto empírico, intuitivo o del sentido común, surgidos de la mera observación de la realidad y sobre la cual se tenga una opinión particular.

Y aunque sería tema para otro artículo, las implicaciones pedagógicas de tomar en cuenta las cuestiones arriba señaladas van en diversos sentidos. Así, por ejemplo, en el diseño de programas de escritura académica que abordan cuestiones que vayan más allá de las consideraciones formales del texto y se trabaje más en hacer evidentes, para los propios alumnos, las estrategias de los escritores expertos, posibilitando así la construcción de un metalenguaje en el novel investigador.

Finalmente, es obligatorio decir que hay más elementos que deben ser observados dentro de los textos escritos, tales como la cohesión léxica y gramatical de los elementos analizados, así como la relación de la problematización y la elaboración de objetivos de investigación y análisis.

\section{Referencias}

Barton, E. L. (1993). "Evidentials, Argumentation, and Epistemological Stance". College English, 55(7), 745-769. National Council of Teachers of English. http://www.jstor.org/stable/378428

Christie, F. y J. R. Martin (eds.) (2008). Language, Knowledge and Pedagogy Functional Linguistic and Sociological Perspectives. Nueva York: Continuum.

García-Córdoba F. y L. T. García-Córdoba (2005). “La problematización. Etapa determinante de una investigación". Cuadernos. México: ISCEEM.

Halliday, M. A. K. (2004). Halliday's Introduction to Functional Grammar. Londres/Nueva York: Routledge.

Hood, S. (2010). Appraising Research: Evaluation in Academic Writing. Nueva York: Palgrave-Macmillan. 
(2010). "Language and Legitimation: Disciplinary Differences in Constructing Space for New Knowledge". Pearling seminar, October 22. hallidaycentre.cityu.edu

Hood, S. y J. R. Martin (2005). “Invocación de actitudes: El juego de la gradación de la valoración en el discurso". Revista Signos, 38(58), 195-220.

Hyland, K. y C. Guinda (eds.) (2012). Stance and Voice in Written Academic Genres. Reino Unido/ Nueva York: Palgrave Macmillan.

Jiménez-Becerra, A. y A. Torres-Carrillo (comps.) (2006). La práctica investigativa en ciencias sociales. Bogotá: Departamento de Ciencias Sociales-Universidad Pedagógica Nacional.

Martin, J. R. (1997). "Analysing Genre: Functional Parameters". En F. Christie y J. R. Martin (eds.). Genre and Institutions: Social Processes in the Workplace and School. Londres: Continuum.

(2000). "Beyond Exchange: Appraisal Systems in English". En S. Hunston y G. Thompson. (eds.). Evaluation in Text. Oxford: Oxford University Press.

(2007). "Construing Knowledge: A Functional Language Perspective". En F. Christie y J. R. Martin (eds.). Language, Knowledge and Pedagogy. Functional Linguistic and Sociological Perspectives. Londres: Continuum.

(2008). Genre Relations Mapping Culture. Londres/Oakville: Equinox.

y D. Rose $(2003,2007)$ Working with Discourse. Second edition. Londres/Nueva York: Continuum.

y P. R. R. White (2005). The Language of Evaluation: Appraisal in English. Gran Bretaña: Palgrave Macmillan.

Pineda-Pineda, I. (2011). "La problematización como registro de posibilidades para la investigación educativa". Multidisciplinas, 10, 115-125. México: UNAM-Acatlán.

Sánchez-Puentes, R. (2014). Enseñar a investigar. Una didáctica nueva de la investigación en ciencias sociales y humanas. México: UNAM.

(1993). "Didáctica de la problematización en el campo científico de la educación". Perfiles Educativos, 61, 64-78. México: Centro de Estudios sobre la Universidad, UNAM/IRESIE/Banco de Datos sobre Educación. 